\title{
Efficacy and Pattern of Antibiotic Usage Among Patients with Cirrhosis and/or Chronic Liver Disease in Telangana, India
}

\author{
Shibnath Kamila ${ }^{1}$, Surampalli Gurunath ${ }^{2 *}$, Nimmagadda Srinivas ${ }^{3}$, \\ Sethu Babu ${ }^{4}$, Giddalur Abhiman ${ }^{1}$, Ravirala Ushasri ${ }^{1}$, Syed Taha ${ }^{1}$, \\ Shreeya Salunke ${ }^{2}$ and Sravan Komittishetty ${ }^{2}$ \\ ${ }^{1}$ Department of Pharmacy Practice, Bharat Institute of Technology, Hyderabad, India; ${ }^{2}$ Department of Pharmacology, \\ Bharat Institute of Technology, Hyderabad, India; ${ }^{3}$ Department of Pharmceutics, Bharat Institute of Technology, Hyderabad, India, \\ ${ }^{4}$ Department Gastroenterology and Hepatology, KIMS Hospital, Secunderabad, Telangana, India
}

\begin{abstract}
Background and objectives: The most common Gram-negative bacteria, such as enteric bacilli, Escherichia coli and Klebsiella pneumoniae, and Gram-positive bacteria, such as Streptococcus spp., are seen in patients suffering from cirrhosis and/or chronic liver diseases. The objective of this prospective observational study was to compare the efficacy and pattern of antibiotic use in patients with bacterial translocation.
\end{abstract}

Methods: This 10-month study was conducted at the Gastroenterology Department of the KIMS hospital, Telangana, India. The patients were more than 18 years of age $(n=60)$ and diagnosed with liver cirrhosis and/ or chronic liver diseases. All data was analyzed statistically, at a significance threshold of $p<0.05$.

Results: Among the 60 patients, the Child-Pugh-Turcotte scores were A in $30 \%$, B in $35 \%$ and C in $14 \%$. White blood cell count was reduced from 12,620 $\pm 1,266$ (before treatment) to 8,385 \pm 944 (after treatment with antibiotics; $p<0.05$ ). Serum glutamic pyruvic transaminase values were reduced from $360.1 \pm 87.3$ (before treatment) to $141.9 \pm 37.9$ (after treatment with antibiotics therapy $(p<0.001)$, whereas serum bilirubin values were reduced from $6.064 \pm 0.91$ (Before treatment) to $3.514 \pm 0.44$ (after treatment with antibiotics therapy; $p<0.0001$ ). The mortality rate was $6.6 \%$, i.e. only 4 patients died post-treatment. It was also observed that meropenem was prescribed in the majority of cases and norfloxacin was the least prescribed of all antibiotics.

Conclusions: Our study suggests that antibiotic treatment might be effective for patients suffering with cirrhosis or chronic liver diseases with improved life expectancy.

Introduction

Liver diseases represent the second largest cause of mortality, with

Keywords: Bacterial translocation; Cirrhosis; Meropenem.

Abbreviations: SBP, spontaneous bacterial peritonitis; BT, bacterial translocation; AEA, appropriate empirical antibiotic; CLD, chronic liver disease; CPT, Child-PughTurcotte; MELD, model for end-stage liver disease; WBC, white blood cell; SGPT, serum glutamic pyruvic transaminase levels.

Received: January 14, 2020; Revised: April 20, 2020; Accepted: May 11, 2020

*Correspondence to: Surampalli Gurunath, Department of Pharmacy Practice, Bharat Institute of Technology, Hyderabad, India.

E-mail: s.gurunath1979@gmail.com

How to cite this article: Kamila S, Gurunath S, Srinivas N, Babu S, Abhiman G, Ushasri R, Taha S, Salunke S, Komittishetty S. Efficacy and Pattern of Antibiotic Usage Among Patients with Cirrhosis and/or Chronic Liver Disease in Telangana, India. Exploratory Research and Hypothesis in Medicine 2020;5(3):97-102. doi: 10.14218/ ERHM.2020.00003. the prevalence of cirrhosis between $5 \%$ and $9 \%$ of the general USA population amongst all digestive diseases, as reported from autopsy studies. The worldwide estimate of mortality from cirrhosis ranked $14^{\text {th }}$ and $10^{\text {th }}$ for cause of death globally and among developed populations respectively, inflicting 771,000 patients. ${ }^{1}$

The definition of cirrhosis is stated as the histological outgrowth of regenerative nodules, with the growth of surrounding fibrous tissues being due to chronic injury to the liver causing end-stage liver disease and portal hypertension. The most common feature noticed in liver cirrhosis patients is the overgrowth of intestinal bacteria, predominantly in the small intestine. The complications associated with cirrhosis with ascites include spontaneous bacterial peritonitis (SBP), occurring via the translocation of gut flora into the mesenteric plexuses and the ascetic fluid contained within. The mortality rate surveyed over 2 years for cirrhosis patients with ascites was estimated to be $50 \%{ }^{2,3}$ However, in patients with ascites, there will be $10-25 \%$ chance of developing SBP, with a sub- 
sequent mortality rate of $30-50 \%$ over a period of 1 year. ${ }^{4,5}$

The pathogenesis for the bacterial infections seen in cirrhosis may be acquired or inherent due to bacterial translocation (BT), intestinal permeability, and interaction of gut microbiota with mesenteric plexuses. Though BT occurs in normal healthy conditions, it is increased in pathological conditions, such as cirrhosis. The primary mechanism for BT may involve the intestinal route to the mesenteric lymph nodes, although other locations of bacterial infections, such as urinary, respiratory tract, etc., may also be responsible. The most common infecting organisms isolated from ascites are Gram-negative enteric bacilli, Escherichia coli, Klebsiella pneumonia and Streptococcus spp. ${ }^{6,7}$

Use of appropriate empirical antibiotic (AEA) ${ }^{8}$ or broad-spectrum antibiotic therapy as empirical treatment was associated with better survival. The current indications of antibiotic prophylaxis in cirrhosis are generally norfloxacin (administered as $400 \mathrm{mg} / 12 \mathrm{~h}$ PO for 7 days); whereas, patients with advanced cirrhosis (defined as at least 2 of the following: ascites, jaundice, hepatic encephalopathy, and malnutrition) receive ceftriaxone (administered as 1 $\mathrm{g}$ /day intravenous for 7 days). Primary prophylaxis of SBP in patients with low protein ascites $(<15 \mathrm{~g} / \mathrm{L})$ is norfloxacin $(400 \mathrm{mg} /$ day PO) or ciprofloxacin $(500 \mathrm{mg} /$ day until liver transplantation $){ }^{8}$

Newer strategies need to be developed for AEA use. The strategies need to track with the local epidemiological issues, such as increase in multidrug resistant infections to aim against empirical antibiotic treatment failure in cirrhosis and improve the survival rate of cirrhotic patients suffering from bactericemia. ${ }^{9}$ Findings from a randomized, controlled clinical trial indicated the combination of meropenem and daptomycin was significantly more effective than ceftazidime in the treatment of nosocomial SBP (86.7 vs. $25 \% ; p<0.001){ }^{10}$

Bacterial infections are frequently noticed in advanced cirrhotic patients, with the altered immunity favoring BT and leading to higher incidences of bacterial infection. It is practically seen that patients with cirrhosis were at higher risk of developing bacterial infection and succumbing to death than those individuals without cirrhosis. Therefore, bacterial infection is a major cause of mortality in cirrhosis patients. Consequently, it is pivotal for early diagnosis and treatment for bacterial infection in the management of cirrhotic patients. The higher survival rate is associated with early initiation of AEA therapy in a heterogeneous patient population suffering from septic shock. However, the available data is very scarce and precludes relating the association between AEA usage and outcome in patients suffering from cirrhosis. Therefore, the authors planned this study to assess the relationship between AEA use and the survival rate of cirrhotic patients with bacteremia.

\section{Materials and methods}

The prospective and observational study was conducted in the Gastroenterology Department of KIMS Hospital, Secunderabad, India with preapproval from the Human Ethics Committee in accordance with ICH-GCP guidelines and Declaration of Helsinki. Written informed consent was obtained from all the patients before enrollment.

Sixty patients of either sex, diagnosed with cirrhosis and chronic liver disease (CLD), such as mild liver infections, hepatitis, bacterial peritonitis, etc., and $>18$ years of age were enrolled for the study. The CLD was evaluated on the basis of alcoholic liver cirrhosis, viral hepatitis $\mathrm{B}$, viral hepatitis $\mathrm{C}$, fever due to unidentified cause, and viral hepatitis with heavy alcoholism. These patients underwent the following examinations: liver function test (elevated gamma-glutamyl transpeptidase and bilirubin, low level of albumin, decreased prothrombin time, hypernatremia and macro-, normo- or microcytic anemia), biopsy, USG abdomen, computed tomography scan, and endoscopy findings. The presence of ascites (proteinaceous fluid 1.5 L) with elevated neutrophils indicated that the patients had infection or BT. Positive culture sensitivity tests or presence of lower gastrointestinal bleeding were also considered. The Child-Pugh-Turcotte (CPT) scores were calculated to assess the risk of mortality in cirrhotic patients.

The exclusion criteria included patients with non-infective cirrhosis or CLD who presented with negative culture sensitivity test, were of age $<18$ years, had upper gastrointestinal bleeding, had been exposed to antibiotic treatment within the past 1 week, were women who were nursing or pregnant, or showed hypersensitivity to the study medication. The patients who were not able to give informed consent or suffering from mental illness that prohibited their ability to give consent were also excluded from the study.

All the relevant and necessary data was collected from in-patient records/MICU case records, lab reports, and prescription records. These data included: CPT and model for end-stage liver disease (MELD) score categories; vitals; liver function test; complete blood profile; investigations like biopsy, USG abdomen, computed tomography scan, endoscopy; culture sensitivity tests.

\section{Assessment}

Efficacy of the antibiotics was inspected by examining parameters like white blood cell (WBC) count and liver function tests, like for measures of serum glutamic pyruvic transaminase levels (SGPT) and bilirubin levels. Analysis of patients based on the WBC and liver function tests was carried out every day. The CPT and MELD scores were originally developed to predict outcomes in cirrhotic patients with portal hypertension and survival (mortality) data of the patients who underwent the elective transjugular intrahepatic portosystemic shunt procedure. MELD scoring is a good predictor for 3-month mortality in a diverse-patient cirrhotic population who were not transjugular intrahepatic portosystemic shunt positioned.

\section{Statistical analysis}

The values were presented as mean \pm standard error of the mean, in absolute numbers and as percentages. Descriptive statistical analysis was used with line and bar diagram representations. All parameters that include SGPT, WBC, serum bilirubin were compared using paired $t$-test with $p<0.05$.

\section{Results}

All the baseline characteristics (mean \pm standard deviation, $n=$ 60; Table 1), such as gender, comorbidities, CPT category, serum bilirubin, and WBC levels, were collected accordingly before treatment. The WBC, serum bilirubin and other elevated levels of the patients were decreased after treatment and the levels showed statistically significant values $(p<0.05)$ at end of treatment.

\section{Baseline characteristics}

The systolic and diastolic blood pressure $(119.1 \pm 14.34,75 \pm$ 
Table 1. Baseline characteristics and clinical data

\begin{tabular}{lccl}
\hline \multirow{2}{*}{ Parameters } & \multicolumn{2}{c}{ Mean \pm standard deviation $n=60$} & \\
\cline { 2 - 4 } & Before treatment & After treatment & - \\
\hline Age in years & & $56.4 \pm 10.2$ & - \\
Male & & $54(90 \%)$ & - \\
Female & & $06(10 \%)$ & $>0.05$ \\
Pulse rate & $82 \pm 7.07$ & $83 \pm 6.45$ & - \\
Alcoholic cirrhosis & $27(45 \%)$ & $27(45 \%)$ & - \\
Chronic liver disease & $33(55 \%)$ & $33(55 \%)$ & - \\
Smoking & $49(81.66 \%)$ & $49(81.66 \%)$ & - \\
Alcohol & $42(70 \%)$ & $42(70 \%)$ & $>0.05$ \\
Systolic blood pressure, $\mathrm{mmHg}$ & $118.8 \pm 20.88$ & $119.1 \pm 14.34$ & $>0.05$ \\
Diastolic blood pressure, $\mathrm{mmHg}$ & $76 \pm 9.12$ & $75 \pm 7.14$ & 0.043 \\
Refractory ascites, $n$ (\%) & $19 \pm 0.4(31.66 \%)$ & $9 \pm 1.3(14.88 \%)$ & 0.038 \\
Abdominal pain, $n$ (\%) & $16 \pm 1.2(26.66 \%)$ & $8 \pm 0.5(13.33 \%)$ & 0.048 \\
Anorexia, $n$ (\%) & $14 \pm 1.01(23.33 \%)$ & $8 \pm 0.3(13.29 \%)$ & $7 \pm 0.34(11.6 \%)$ \\
Fever, $n$ (\%) & $12 \pm 0.71(20 \%)$ & $5 \pm 0.21(8.25 \%)$ & 0.031 \\
Melena, $n$ (\%) & $9 \pm 0.21(15 \%)$ & $6 \pm 0.5(9.79 \%)$ & 0.043 \\
Swelling of leg, $n$ (\%) & $14 \pm 1.03(23.33 \%)$ & & 0.037 \\
\hline
\end{tabular}

7.14) post-treatment showed no significant changes and were similar to the values obtained prior the treatment, i.e. $118.8 \pm 20.88$, $76 \pm 9.12$ respectively. The results of refractory ascites, anorexia, swelling of leg, fever, melena, and abdominal pain after treatment (i.e. $31.66 \%, 13.29 \%, 9.79 \%, 11.6 \%, 8.25 \%$, and $13.33 \%$ respectively) significantly $(p<0.05)$ reduced to almost half of the values from before treatment (i.e. $14.88 \%, 23.33 \%, 23.33 \%, 20 \%, 15 \%$, and $26.66 \%$ respectively).

\section{WBC count}

Our study noticed that the mean WBC count was reduced from $12,620 \pm 1,266$ (before treatment) to 8,385 \pm 944 (after treatment with antibiotics, $p<0.05$; Fig. 1a).

\section{SGPT levels}

SGPT values were reduced after treatment with antibiotics therapy $(p<0.001$; Fig. 1b).

\section{Serum bilirubin}

In addition, serum bilirubin values were also reduced after treatment with antibiotics therapy ( $p<0.0001$; Fig. 1c) when compared to the pre-treatment values.

\section{Percentage of people with various CPT classes}

The outcome of CPT scores in various patient populations is depicted in Figure 2, which shows CPT-A as 30\% with improved outcomes, CTP-B as 35\% with partially improved outcomes, and CTP-C as $14 \%$ representing totally relieved outcomes from cirrhosis with antibiotic regimen treatment.

\section{Prescribing pattern of antibiotics}

Meropenem was prescribed to the majority of patients and norfloxacin was the least prescribed of all antibiotics (Fig. 3).

\section{Assessment of MELD}

Only four patients died in our study, giving a mortality percentage of $6.6 \%$, and the rest survived and recovered, implying that antibiotic treatment was a good indicator as the MELD scores were reduced to $<9$.

\section{Percentages of causative organisms}

About $12.5 \%$ of patients were infected with Klebsiella, $7.5 \%$ of patients were infected with $E$. coli, and about $5 \%$ of patients with Streptococcus.

\section{Discussion}

The pathogenesis of cirrhosis involves regenerative nodular development with the formation of fibrous tissues due to chronic liver injury causing end-stage liver disease and portal hypertension. ${ }^{1}$ Cirrhosis can be asymptomatic and it may be identified only when the resulting effects of liver complications arise. ${ }^{11-15}$ The $\mathrm{CPT}$ (modified) scoring system is widely employed to foresee the 

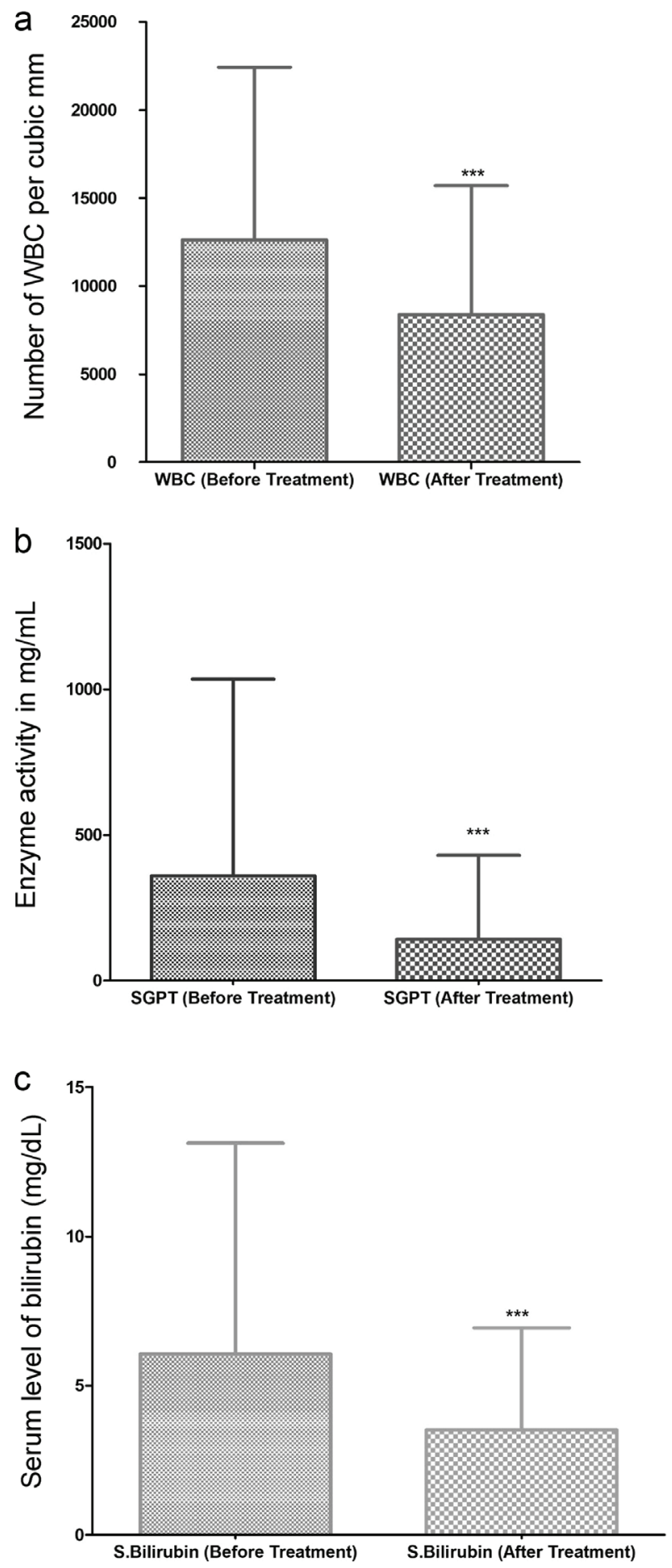

Fig. 1. Depiction of changed levels of WBC count, SGPT, and serum bilirubin in patients with cirrhosis and CLD before and after antibiotic treatment. (a) White blood cell count (WBC), (b) Serum glutamic pyruvic transaminase levels (SGPT), (c) Serum bilirubin levels. CLD, Chronic liver diseases. ${ }^{* * *} p<0.001$.

prognosis of cirrhosis and its complications in the liver disease patient population. ${ }^{16}$ Ascites occurs in $50 \%$ of patients suffering from cirrhosis for a period of 10 years and is the most common

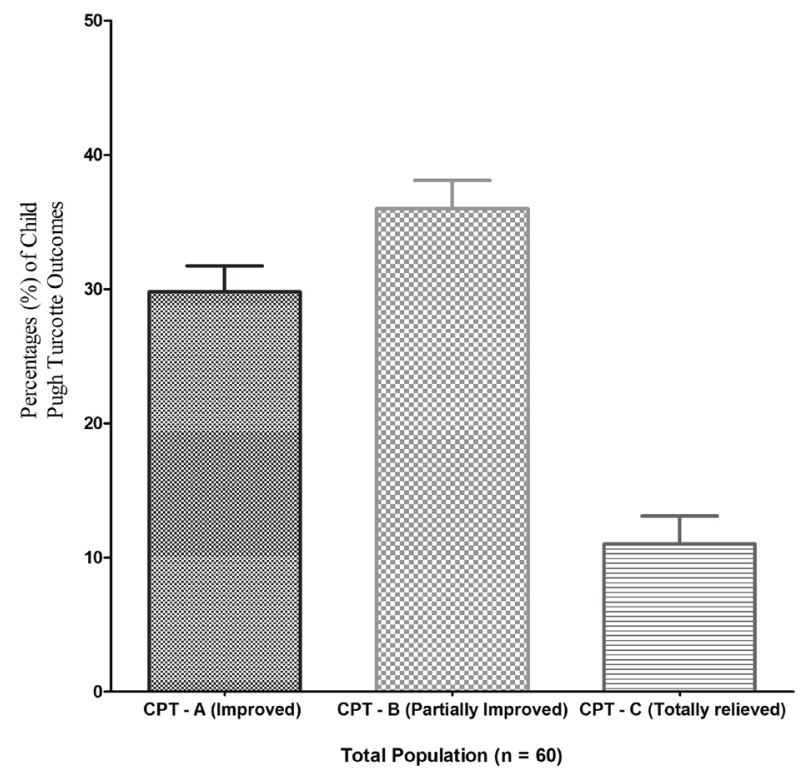

Fig. 2. Analysis of class A, B, and C patients based on the treatment outcomes involving percentages of various populations suffering from ChildPugh-Turcotte.

portal hypertension complication with a mortality rate of $50 \%$ over 2 years. ${ }^{17-19}$

SBP occurs as an ascitic fluid infection, without any intra-abdominal pathogenesis. About $10-30 \%$ of SBP patients get admitted with ascites. ${ }^{20-21}$ Most of the cultural sensitivity tests $(50 \%$ to $60 \%$ ) involve the isolation of infection-inducing-form of the ascetic fluid or blood cultures. ${ }^{20-21}$ The most frequent organisms isolated from the tissue cultures were E. coli $(70 \%)$, Klebsiella species (10\%), Proteus and Streptococcus species, along with Enterococcus faecalis (4\% each organism). ${ }^{20}$

Most of the trials we reviewed that reported on a decrease in SBP incidence did not show the impact of prophylactic antibiotic therapy on mortality. But, it was observed that underlying liver dysfunction played a greater role in mortality than SBP. ${ }^{22-25}$ Along with liver disease, renal impairment could be another prognostic cause for mortality over SBP, but as the study was poorly designed it is difficult to highlight the definite cause. ${ }^{26}$

Most of the studies included patients based on their long-term treatment modality involving primary and secondary prophylaxis, with unpredictable risk factors for SBP. This mixed patient population made it difficult to discover which group of patients received the most benefit from prophylactic antibiotic use. Therefore, our findings clearly indicated that meropenem, metronidazole, rifaximin and piperacillin/tazobactam had a significant impact in reducing CPT and MELD scores along with WBC count, SGPT and serum bilirubin levels (Fig. 1) in treating liver cirrhosis, when compared to other antibiotic combinations and their usage in treatment regimen of CLD (Fig. 3).

Although several studies include the use of antibiotic prophylaxis for preventing SBP, none evaluate the appropriate time to start the regimen. In most of the studies, it was noticed that the patients enrolled were often hospitalized and later found to have low levels of ascitic fluid proteins, at which the antibiotic prophylactic regimen was initiated. ${ }^{27-28}$ The first episode probability for a patient with cirrhosis and ascites to develop SBP has been estimated to be between $11 \%$ and $27 \%$. Once a patient develops SBP, the incidence of a second episode is as much as $70 \%$. Currently, 


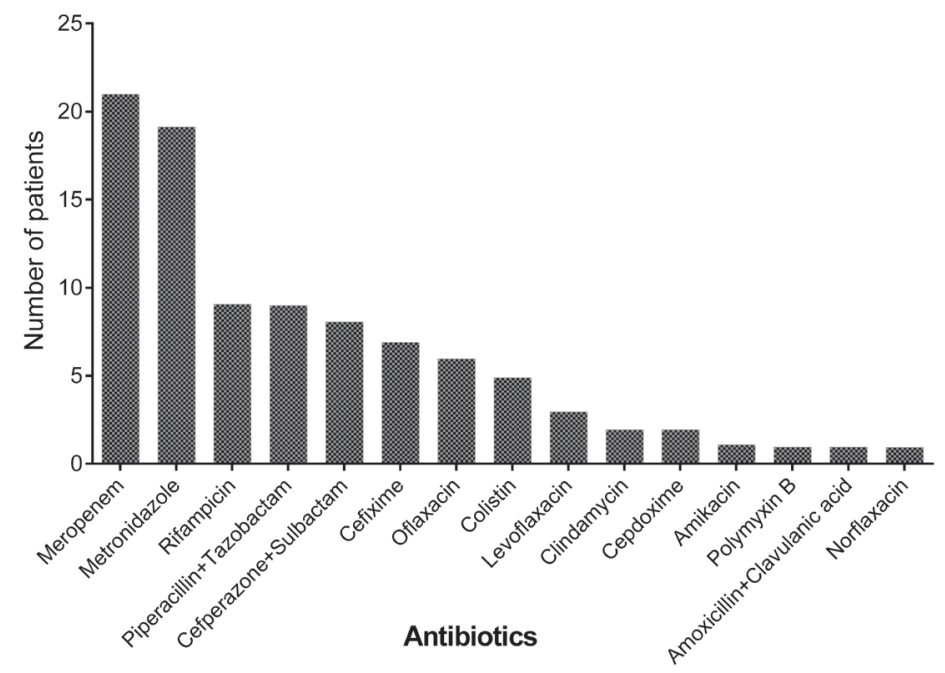

Fig. 3. Pattern and types of antibiotics prescribed in patients with cirrhosis.

there is very little information on hand about the risks associated with the long-term use of antibiotics, such as resistance patterns, Clostridium difficile infection, and other adverse effects. Due to development of bacterial resistance, prophylaxis should be limited to patients with higher risk of SBP.

BT is an immune deficiency that may be acquired or caused by genetic susceptibility and functions in tandem as a pathophysiological perpetrator for most cirrhosis-related bacterial infections. ${ }^{29} \mathrm{BT}$ exists in healthy conditions but is elevated in cirrhosis, and therefore is considered to be pathological BT. Nevertheless, studies on the relationship between antibiotic therapy and BT are rare, but in a study by Wen et al. ${ }^{29}$ conducted on the frequent use of antibiotics and the effect of broad-spectrum antibiotics on BT in experimental rat models of burn or sepsis injury, it was observed that broad-spectrum antibiotics promote BT in burned rats but prevent BT in septic rats, thereby preventing the spread of BT to distant organs, such as the liver and lungs. In agreement with those findings, our study outcomes clearly indicated that early initiation of AEA therapy led to a higher survival rate. Our findings in the current study are unique and no studies so far have reported the association between antibiotic therapy and BT in a cirrhotic population with a similar treatment regimen employed in our study.

Our study was conducted on the "efficacy and pattern of antibiotic usage among the patients with cirrhosis and/or chronic liver disease in Telangana, India" admitted to KIMS hospital with a sample size of 60 , studied over a period of 6 months. CLD is seen mostly in the age group between 51 and 60 years and is more common in males than females. All the subjects who used antibiotics have shown reduced MELD, CPT, WBC, SGPT and serum bilirubin levels after the completion of antibiotic therapy. In accordance to other studies, CPT and MELD scores have shown good outcomes in our findings upon antibiotic treatment regimen, thus indicating that CPT and MELD scoring systems can be used to widely assess the prognosis of liver cirrhosis. ${ }^{30}$

Efficacy of the antibiotics was inspected by studying the parameters like WBC test, liver function tests (i.e. SGPT) and serum bilirubin levels. The widely used antibiotics are meropenem, metronidazole, rifaximin and piperacillin/tazobactam. Eighty percent of the patients received multiple antibiotic therapies more than one antibiotic during their stay in MICU. Only four patients died during the treatment and the mortality rate was found to be $6.6 \%$.
Among 60 subjects, antibiotics were found to be effective in $93 \%$ of the total subjects, and $7 \%$ of the patients died due to unknown etiologies and/or due to underlying disease conditions or comorbidities.

\section{Future directions}

The studies on liver cirrhosis will be planned with greater sample size. This design rules out the various type of antibiotics used in wider patient population at different centers involving modified CPT and MELD scoring points. Futher these studies will also highlight major efficacy parameters used for liver cirrhosis diseases in India.

\section{Conclusions}

Our findings suggest that patients suffering with ailments such as cirrhosis or chronic liver disease, when treated with antibiotics, show effective improvement in various clinical parameters along with an improvement in their life expectancy. Further study is required to understand the exact rate of efficacy with larger sample size.

\section{Acknowledgments}

None.

Data sharing statement

No additional data is available.

\section{Funding}

The study has not received any funding or grants and was carried out without any financial support. 


\section{Conflict of interest}

None.

\section{Author contributions}

Study design and performance of experiments (SKa, SB, GA, RU, $\mathrm{ST}$ ), analysis and interpretation of data (SKa, SB, GA, RU, ST), manuscript writing (SKa, SG), critical revision, statistical analysis (SG, NS, SS, SKo), administration, technical or material support (SB).

\section{References}

[1] Sandler RS, Everhart JE, Donowitz M, Adams E, Cronin K, Goodman $C$, et al. The burden of selected digestive diseases in the United States. Gastroenterology 2002;122(5):1500-1511. doi:10.1053/ gast.2002.32978.

[2] Ginès $P$, Martin PY, Neiderberger $M$. Prognostic significance of renal dysfunction in cirrhosis. Kidney Int Suppl 1997;61:S77-82.

[3] Suzuki K, Takikawa Y. Marked improvement in the resolution of, and survival rates in, spontaneous bacterial peritonitis. J Gastroenterol 2002;37(2):149-150. doi:10.1007/s005350200014.

[4] Runyon BA. Spontaneous bacterial peritonitis: an explosion of information. Hepatology 1988;8(1):171-175. doi:10.1002/hep.1840080131.

[5] Garcia-TSAO G. Identifying new risk factors for spontaneous bacterial peritonitis: how important is it? Gastroenterology 1999;117(2):495499. doi:10.1053/gast.1999.0029900495.

[6] Mowat C, Stanley AJ. Review article: spontaneous bacterial peritonitis-diagnosis, treatment and prevention. Aliment Pharmacol Ther 2001;15:1851-1859. doi:10.1046/j.1365-2036.2001.01116.x.

[7] Parsi MA, Atreja A, Zein NN. Spontaneous bacterial peritonitis: recent data on incidence and treatment. Cleve Clin J Med 2004;71(7):569576. doi:10.3949/ccjm.71.7.569.

[8] Park H, Jang KJ, Jang W, Park SH, Park JY, Jeon TJ, et al. Appropriate empirical antibiotic use and 30-d mortality in cirrhotic patients with bacteremia World J Gastroenterol. 2015;21(12):3587-3592. doi:10.3748/wjg.v21.i12.3587.

[9] Merli M, Lucidi C, Di Gregorio V, Lattanzi B, Giannelli V, Giusto M, et al. An empirical broad spectrum antibiotic therapy in health-care-associated infections improves survival in patients with cirrhosis: A randomized trial. Hepatology 2016;63(5):1632-1639. doi:10.1002/hep.28332.

[10] Piano S, Fasolato S, Salinas F, Romano A, Tonon M, Morando F, et al. The empirical antibiotic treatment of nosocomial spontaneous bacterial peritonitis: Results of a randomized, controlled clinical trial. Hepatology 2016;63(4):1299-1309. doi:10.1002/hep.27941.

[11] Schuppan D, Afdahl NH. Liver cirrhosis. Lancet 2008;371:838-851. doi:10.1016/\$0140-6736(08)60383-9.

[12] Dib N, Oberti F, Calès P. Current management of the complications of portal hypertension: variceal bleeding and ascites. Canadian Medical Association Journal 2006;174(10):1433-1443. doi:10.1503/ cmaj.051700.

[13] Kuiper JJ, de Man RA, van Buuren HR. Review article: management of ascites and associated complications in patients with cirrhosis. Aliment Pharmacol Ther 2007;26(suppl 2):183-193. doi:10.1111/ j.1365-2036.2007.03482.x.

[14] Sandhu BS, Sanyal AJ. Management of ascites in cirrhosis. Clin Liver Dis 2005;9(4):715-732. doi:10.1016/j.cld.2005.07.008.

[15] Sanyal AJ, Bosch J, Blei A, Arroyo V. Portal hypertension and its complications. Gastroenterology 2008;134(6):1715-1728. doi:10.1053/j. gastro.2008.03.007.

[16] Pugh RN, Murray-Lyon IM, Dawson JL, Pietroni MC, Williams R. Transection of the oesophagus for bleeding oesophageal varices. $\mathrm{Br} \mathrm{J}$ Surg 1973;60(8):646-649. doi:10.1002/bjs.1800600817.

[17] Moore KP, Wong F, Gines P, Bernardi M, Ochs A, Salerno F, et al. The management of ascites in cirrhosis: report on the consensus conference of the International Ascites Club. Hepatology 2003;38(1):258266. doi:10.1053/jhep.2003.50315.

[18] Choudhury J, Sanyal AJ. Treatment of ascites. Cur Treat Options Gastroenterology 2003;6(6):481-491. doi:10.1007/s11938-003-0050-5.

[19] Runyon BA. Care of patients with ascites. N Engl J Med 1994;330(5):337-342. doi:10.1056/NEJM199402033300508.

[20] Koulaouzidis A, Bhat S, Karagiannidis A, Tan WC, Linaker BD. Spontaneous bacterial peritonitis. Postgrad Med J 2007;83(980):379-383. doi:10.1136/pgmj.2006.056168.

[21] Blei AT. Portal hypertension and its complications. Curr Opin Gastroenterol 2007;23(3):275-282. doi:10.1097/MOG.0b013e3280b0841f.

[22] Ginés P, Rimola A, Planas R, Vargas V, Marco F, Almela M, et al. Norfloxacin prevents spontaneous bacterial peritonitis recurrence in cirrhosis: results of a double-blind, placebo-controlled trial. Hepatology 1990;12(4 Pt 1):716-724. doi:10.1002/hep.1840120416.

[23] Novella M, Solà R, Soriano G, Andreu M, Gana J, Ortiz J, et al. Continuous versus inpatient prophylaxis of the first episode of spontaneous bacterial peritonitis with norfloxacin. Hepatology 1997;25(3):532536. doi:10.1002/hep.510250306.

[24] Rolachon A, Cordier L, Bacq Y, Nousbaum JB, Franza A, Paris JC, et al. Ciprofloxacin and long-term prevention of spontaneous bacterial peritonitis: results of a prospective controlled trial. Hepatology 1995;22(4 Pt 1):1171-1174. doi:10.1016/0270-9139(95)90626-6.

[25] Fernández J, Navasa M, Planas R, Montoliu S, Monfort D, Soriano G, et al. Primary prophylaxis of spontaneous bacterial peritonitis delays hepatorenal syndrome and improves survival in cirrhosis. Gastroenterology 2007;133(3):818-24. doi:10.1053/j.gastro.2007.06.065.

[26] Singh N, Gayowski T, Yu VL, Wagener MM. Trimethoprim-sulfamethoxazole for the prevention of spontaneous bacterial peritonitis in cirrhosis: a randomized trial. Ann Intern Med 1995;122(8):595-598. doi:10.7326/0003-4819-122-8-199504150-00007.

[27] Llach J, Rimola $A$, Navasa $M$, Ginès $P$, Salmerón JM, Ginès $A$, et al. Incidence and predictive factors of first episode of spontaneous bacterial peritonitis in cirrhosis with ascites: relevance of ascites fluid protein concentration. Hepatology 1992;16(3):724-727. doi:10.1002/ hep. 1840160318

[28] Andreu M, Sola R, Sitges-Serra A, Alia C, Gallen M, Vila MC, et al. Risk factors for spontaneous bacterial peritonitis incirrhotic patients with ascites. Gastroenterology 1993;104:1133-1138. doi:10.1016/00165085(93)90284-J.

[29] Wen ZL, Zhang LD, Liu SZ, Liu J, Chen YZ, Chen DC. Effect of broadspectrum antibiotics on bacterial translocation in burned or septic rats. Chin Med J (Engl) 2019;132(10):1179-1187. doi:10.1097/ CM9.0000000000000242.

[30] Peng Y, Qi X, Guo X. Child-Pugh Versus MELD Score for the Assessment of Prognosis in Liver Cirrhosis: A Systematic Review and Meta-Analysis of Observational Studies. Medicine (Baltimore) 2016;95(8):e2877. doi:10.1097/MD.0000000000002877. 